\title{
Deconfined quantum criticality in the two dimensional Kondo lattice model
}

\author{
Ki-Seok Kim \\ School of Physics, Korea Institute for Advanced Study, Seoul 130-012, Korea
}

(Dated: November 20, 2018)

\begin{abstract}
We investigate the continuous quantum phase transition from an antiferromagnetic metal to a heavy fermion liquid based on the Kondo lattice model in two dimensions. We propose that antiferromagnetic spin fluctuations and conduction electrons fractionalize into neutral bosonic spinons and charged spinless fermions at the quantum critical point. This deconfined quantum criticality leads us to establish a critical field theory in terms of the fractionalized fields interacting via emergent $\mathrm{U}(1)$ gauge fields. The critical field theory not only predicts non-Fermi liquid physics near the quantum critical point but also recovers Fermi liquid physics away from the quantum critical point.
\end{abstract}

PACS numbers: 71.10.-w, 71.10.Hf, 71.27.+a, 75.30.Mb

Nature of quantum criticality is one of the central interests in modern condensed matter physics. Especially, deconfined quantum criticality has been proposed in various strongly correlated electron systems such as low dimensional quantum antiferromagnetism 1,2 , 3, 3, 5, 6, 7, 8, 9] and heavy fermion liquids [10, 11, 12, 13]. In the present paper we focus our attention on the quantum phase transition from an antiferromagnetic metal to a heavy fermion liquid in the two dimensional Kondo lattice model. In the Landau-Ginzburg-Wilson theoretical frame work the quantum phase transition would belong to the first order because the two phases are characterized by two different order parameters [1]. However, it is well known that there exists a continuous quantum phase transition between the two phases 10, 11, 12, 13]. In this paper we propose that the continuous quantum phase transition can be realized via deconfined quantum criticality, where antiferromagnetic spin fluctuations and conduction electrons fractionalize into neutral bosonic spinons and charged spinless fermions at the quantum critical point. Near the quantum critical point two kinds of critical fluctuations are expected to arise. One would correspond to critical fluctuations of Kondo singlets and the other, critical antiferromagnetic spin fluctuations. Remarkably, the emergent spinless fermions are associated with critical fluctuations of the Kondo singlets while the critical bosonic spinons result from critical spin fluctuations.

We consider the Kondo lattice model in two dimensions

$$
\begin{aligned}
& H=H_{c}+H_{m}+H_{K}, \\
& H_{c}=-t \sum_{<i, j>} c_{i \sigma}^{\dagger} e^{i A_{i j}} c_{j \sigma}-h . c ., \\
& H_{m}=J \sum_{<i, j>} \vec{S}_{i} \cdot \vec{S}_{j}, \\
& H_{K}=J_{K} \sum_{i} \vec{S}_{i} \cdot c_{i \sigma}^{\dagger} \vec{\tau}_{\sigma \sigma^{\prime}} c_{i \sigma^{\prime}} .
\end{aligned}
$$

$H_{c}$ describes dynamics of conduction electrons $c_{i \sigma}$ and $H_{m}$, that of local spins $\vec{S}_{i}$. Here $t$ is a hopping integral of the conduction electrons and $J$, an antiferromagnetic exchange coupling of the local spins. $A_{i j}$ is an external electromagnetic vector potential in a lattice version. $H_{K}$ represents Kondo couplings between the conduction and localized spins, where $J_{K}$ is a Kondo coupling constant.

We perform the Haldane mapping for $H_{m}$ to derive the $\mathrm{O}(3)$ nonlinear $\sigma$ model as a low energy effective field theory [14, 15]. In this mapping high energy antiferromagnetic spin fluctuations would induce new interactions between low energy spin degrees of freedom and conduction electrons. We can derive the following expression for the Kondo lattice model

$$
\begin{aligned}
& Z=\int D c_{i \sigma} D \vec{n}_{i} \delta\left(\left|\vec{n}_{i}\right|^{2}-1\right) e^{-S_{m}-S_{K}-S_{c},} \\
& S_{m}=i S \sum_{i}(-1)^{i} \int_{0}^{c \beta} d x_{0} \int_{0}^{1} d u \vec{n}_{i} \cdot\left(\frac{\partial \vec{n}_{i}}{\partial u} \times \frac{\partial \vec{n}_{i}}{\partial x_{0}}\right) \\
& +\int_{0}^{c \beta} d x_{0}\left[\frac{1}{2 g} \sum_{i}\left|\partial_{0} \vec{n}_{i}\right|^{2}-\frac{1}{g} \sum_{<i, j>} \vec{n}_{i} \cdot \vec{n}_{j}\right], \\
& S_{K}=-i \frac{2 a^{d} J_{K}}{c g} \sum_{i} \int_{0}^{c \beta} d x_{0}\left(\frac{\partial \vec{n}_{i}}{\partial x_{0}} \times \vec{n}_{i}\right) \cdot c_{i \sigma}^{\dagger} \vec{\tau}_{\sigma \sigma^{\prime}} c_{i \sigma^{\prime}} \\
& +\frac{2 \sqrt{2 d} a^{d-1} J_{K}}{c g} \sum_{i} \int_{0}^{c \beta} d x_{0}(-1)^{i} \vec{n}_{i} \cdot c_{i \sigma}^{\dagger} \vec{\tau}_{\sigma \sigma^{\prime}} c_{i \sigma^{\prime}}, \\
& S_{c}=\int_{0}^{c \beta} d x_{0}\left[\sum_{i} c_{i \sigma}^{\dagger}\left(\partial_{0}-i A_{i 0}-\mu\right) c_{i \sigma}\right. \\
& \left.-t_{c} \sum_{<i, j>} c_{i \sigma}^{\dagger} e^{i A_{i j}} c_{j \sigma}-h . c .-\frac{a^{2 d} J_{K}^{2}}{c^{2} g} \sum_{i}\left|c_{i \sigma}^{\dagger} \vec{\tau}_{\sigma \sigma^{\prime}} c_{i \sigma^{\prime}}\right|^{2}\right] .
\end{aligned}
$$

The local spins are described by the $\mathrm{O}(3)$ nonlinear $\sigma$ model of a low energy spin variable $\vec{n}$, where $g=$ $\frac{2 \sqrt{2 d}}{S} a^{d-1}$ is a spin stiffness parameter $\left(g^{-1}\right)$ and $c=$ $\sqrt{2 d} J S a$, a velocity of spin waves 14, 15]. $a$ is a lattice spacing, $S$, the value of localized spins, and $d$, a spatial dimension. Here $S=1 / 2$ and $d=2$. The first term in $S_{m}$ is a Berry phase action, where $u$ and $x_{0}$ are two parameters in a unit sphere 14, 15]. $x_{0}=c \tau$ is considered to be a rescaled time. The mapping to the nonlinear $\sigma$ model results in nontrivial couplings between the low energy spins and the conduction electrons. The first term 
in $S_{K}$ arises from high energy spin fluctuations while the second originates from the usual Kondo couplings. Notice $(-1)^{i}$ in the second term. In the action $S_{c}$ for the conduction electrons $\mu$ is a chemical potential of the conduction electrons and $A_{0 i}$, an external Coulomb potential. The hopping integral is redefined to be $t_{c}=t / c$. The last term in $S_{c}$ represents local ferromagnetic interactions for the conduction electrons, originating from the contribution of high energy spin fluctuations in the Haldane mapping.

The next step would be to integrate over high energy conduction electrons. It is not easy to integrate over the conduction electrons. Considering our objective to establish a critical field theory in terms of the fractionalized fields, we are satisfied with estimating possible effects of the Kondo couplings. The second Kondo coupling term in $S_{K}$ would reduce the spin stiffness $g^{-1}$. In the absence of the Kondo couplings the nonlinear $\sigma$ model exhibits an antiferromagnetic long range order 14, 15]. It is well known that the $\mathrm{O}(3)$ nonlinear $\sigma$ model shows a continuous quantum phase transition from antiferromagnetism to paramagnetism at a nonzero critical spin stiffness, $g_{c}^{-1}$ in two space and one time dimensions [14, 15]. Thus, increasing the Kondo coupling constant $J_{K}$ may drive the spin stiffness $g^{-1}$ to the critical value $g_{c}^{-1}$, causing the magnetic quantum phase transition. We view the continuous quantum phase transition in the Kondo lattice model as the magnetic one induced by the Kondo interactions. Furthermore, the first Kondo coupling term in $S_{K}$ would affect the Berry phase action in $S_{m}$. It is well known that in two leg ladders the contribution of Berry phase cancels between the legs 16, 17]. The same mechanism works in the double layered quantum antiferromagnets 2, 18]. The presence of the Kondo couplings with the conduction electrons are expected to weaken the contribution of Berry phase.

Recently, it was argued that at the quantum critical point of the $O(3)$ nonlinear $\sigma$ model, spin 1 critical antiferromagnetic fluctuations break up into more elementary spin $1 / 2$ critical bosonic excitations called spinons 1, 2, 3]. This is the precise meaning of deconfined quantum criticality in the quantum antiferromagnetism. In the present paper we apply the deconfined quantum criticality in the magnetic quantum phase transition to the present Kondo lattice model because the quantum phase transition in the Kondo lattice model is conjectured to be a magnetic transition. This idea can be realized in the $C P^{1}$ representation $\vec{n}=\frac{1}{2} z_{\sigma}^{\dagger} \vec{\tau}_{\sigma \sigma^{\prime}} z_{\sigma^{\prime}}$, where $z_{\sigma}$ is a bosonic spinon 1, 2, 3, 14, 15]. A problem is the fate of conduction electrons near the quantum critical point. In order to investigate this we focus on the second Kondo coupling term $\vec{n}_{i} \cdot c_{i \sigma}^{\dagger} \vec{\tau}_{\sigma \sigma^{\prime}} c_{i \sigma^{\prime}}$ in $S_{K}$. Using $\vec{n} \cdot \vec{\tau}=U \tau^{3} U^{\dagger}$ with an $\mathrm{SU}(2)$ matrix $U=\left(\begin{array}{cc}z_{\uparrow} & -z_{\downarrow}^{\dagger} \\ z_{\downarrow} & z_{\uparrow}^{\dagger}\end{array}\right)$, we can represent the Kondo interaction term as $c_{i \sigma}^{\dagger}\left(U_{i} \tau^{3} U_{i}^{\dagger}\right)_{\sigma \sigma^{\prime}} c_{i \sigma^{\prime}}$. This term can be solved by introducing the following gauge transformation $\chi_{\sigma}=U_{\sigma \sigma^{\prime}}^{\dagger} c_{\sigma^{\prime}}$. The components of the $\chi_{\sigma}$ field are given by $\chi_{\sigma}=\left(\begin{array}{c}\chi_{\uparrow} \\ \chi_{\downarrow}\end{array}\right)=\left(\begin{array}{c}z_{\uparrow}^{\dagger} c_{\uparrow}+z_{\downarrow}^{\dagger} c_{\downarrow} \\ -z_{\downarrow} c_{\uparrow}+z_{\uparrow} c_{\downarrow}\end{array}\right)$, where the $\chi_{\uparrow}$ field represents the usual Kondo hybridization and the $\chi_{\downarrow}$ field denotes the singlet pairing between the bosonic spinon and the conduction electron. The $\chi_{\sigma}$ field can be considered to express Kondo resonances. Near the quantum critical point the bosonic spinons $U_{\sigma \sigma^{\prime}}^{\dagger}$ emerging from localized spins are expected to hybridize with the conduction electrons $c_{\sigma^{\prime}}$ via the Kondo interactions, resulting in charged spinless fermions $\chi_{\sigma}$. Another way to say this is that the conduction electrons $c_{\sigma}$ fractionalize into the bosonic spinons $U_{\sigma \sigma^{\prime}}$ and the charged spinless fermions $\chi_{\sigma}$, i.e., $c_{\sigma}=U_{\sigma \sigma^{\prime}} \chi_{\sigma^{\prime}}$ owing to the Kondo interactions.

Representing Eq. (2) in terms of the fractionalized fields, $U_{\sigma \sigma^{\prime}}$ and $\chi_{\sigma}$, we can obtain the following expression

$$
\begin{aligned}
& Z=\int D \chi_{\sigma} D U_{\sigma \sigma^{\prime}} e^{-S_{c}-S_{m}} \\
& S_{c}=\int d^{3} x\left[\chi _ { \sigma } ^ { \dagger } \left(\left[\partial_{0}-i A_{0}\right] \delta_{\sigma \sigma^{\prime}}+\left[U^{\dagger} \partial_{0} U\right]_{\sigma \sigma^{\prime}}\right.\right. \\
& \left.\left.+\vec{v}_{F} \cdot\left(\left[i \vec{\nabla}+\vec{k}_{F}+\vec{A}\right] \delta_{\sigma \sigma^{\prime}}+i\left[U^{\dagger} \vec{\nabla} U\right]_{\sigma \sigma^{\prime}}\right)\right) \chi_{\sigma^{\prime}}\right] \\
& +\frac{2 \sqrt{2 d} a^{d-1} J_{K}}{c \tilde{g}} \int_{0}^{c \beta} d x_{0} \sum_{k} \chi_{k \sigma}^{\dagger} \tau_{\sigma \sigma^{\prime}}^{3} \chi_{k+Q \sigma^{\prime}} \\
& S_{m}=\int d^{3} x\left[\operatorname{Tr}\left(\frac{1}{4 \tilde{g}}\left|\partial_{\mu}\left(U \tau^{3} U^{\dagger}\right)\right|^{2}\right)\right] .
\end{aligned}
$$

Notice that the bare spin stiffness $g^{-1}$ is assumed to renormalize into $\tilde{g}^{-1}$, resulting from the contribution of high energy conduction electrons via the Kondo couplings. In the action $S_{c}$ for the conduction electrons we performed the continuum limit near the Fermi surface. Here $v_{F}$ is a Fermi velocity and $k_{F}$, a Fermi wave vector. The main point in $S_{c}$ is that the effect of couplings between antiferromagnetic spin fluctuations and conduction electrons appears in the kinetic energy of the $\chi_{\sigma}$ field via the gauge transformation $\chi_{\sigma}=U_{\sigma \sigma^{\prime}}^{\dagger} c_{\sigma^{\prime}}$. The last term in $S_{c}$ originating from the Kondo couplings now represents the contribution of Zeeman energy under staggered magnetic fields, where $Q$ is a momentum $(\pi, \pi)$. The staggered Zeeman term splits the two Kondo resonance states. In the limit of large Zeeman splitting the $\chi_{\uparrow}$ fields describe the Kondo resonances at odd sites while the $\chi_{\downarrow}$ fields do at even sites. In the $\mathrm{O}(3)$ nonlinear $\sigma$ model we ignored the Berry phase action because the first Kondo coupling term of $S_{K}$ in Eq. (2) is expected to weaken the contribution of Berry phase as the case of the double layered quantum antiferromagnets 2, 16, 17, 18]. The first Kondo coupling term of $S_{K}$ in Eq. (2) is also neglected. This term does not affect critical phenomena because the bare scaling dimension of $\left(\frac{\partial \vec{n}_{i}}{\partial x_{0}} \times \vec{n}_{i}\right) \cdot c_{i \sigma}^{\dagger} \vec{\tau}_{\sigma \sigma^{\prime}} c_{i \sigma^{\prime}}$ is larger than 3 . Thus, the scaling dimension of the coupling constant $\frac{a^{d} J_{K}}{c g}$ is negative, indicating that the couplings vanish in the low energy limit. The local ferromagnetic interactions of $S_{c}$ in Eq. (2) can be also ignored in the 
low energy limit. In the spinon representation this term is $-\frac{a^{2 d} J_{K}^{2}}{c^{2} g}\left|c_{\sigma}^{\dagger} \vec{\tau}_{\sigma \sigma^{\prime}} c_{\sigma^{\prime}}\right|^{2}=-\frac{a^{2 d} J_{K}^{2}}{c^{2} \tilde{g}}\left|\chi_{\sigma}^{\dagger}\left(U^{\dagger} \vec{\tau}_{\sigma \sigma^{\prime}} U\right)_{\sigma \sigma^{\prime}} \chi_{\sigma^{\prime}}\right|^{2}$. Since the scaling dimension of $\left|\chi_{\sigma}^{\dagger}\left(U^{\dagger} \vec{\tau}_{\sigma \sigma^{\prime}} U\right)_{\sigma \sigma^{\prime}} \chi_{\sigma^{\prime}}\right|^{2}$ is much larger than 3 , this term vanishes in the low energy limit.

The effective action Eq. (3) has not only the trivial electromagnetic $U_{A}(1)$ gauge symmetry but also a new emergent $U_{a}(1)$ gauge symmetry. This $U_{a}(1)$ gauge symmetry guarantees the invariance of the action Eq. (3) under the gauge transformations of

$$
\begin{aligned}
& \chi_{\sigma}^{\prime}=\left[e^{i \vartheta \tau^{3}}\right]_{\sigma \sigma^{\prime}} \chi_{\sigma^{\prime}}, \quad U_{\sigma \sigma^{\prime}}^{\prime}=U_{\sigma \alpha}\left[e^{-i \vartheta \tau^{3}}\right]_{\alpha \sigma^{\prime}}, \\
& c_{\sigma}^{\prime}=c_{\sigma}, \quad A_{\mu}^{\prime}=A_{\mu} .
\end{aligned}
$$

This local gauge symmetry implies that there should be a new emergent $\mathrm{U}(1)$ gauge field corresponding to the gauge symmetry. Indeed, performing some standard algebra such as the Hubbard-Stratonovich transformation [19], we can see the emergence of new $\mathrm{U}(1)$ gauge fields $a_{\mu}$

$$
\begin{aligned}
& Z_{U}=\int D U_{\sigma \sigma^{\prime}} \exp \left[-\int d^{3} x\left(\operatorname{Tr}\left(\frac{1}{4 \tilde{g}}\left|\partial_{\mu}\left(U \tau^{3} U^{\dagger}\right)\right|^{2}\right)\right.\right. \\
& \left.\left.+\chi_{\sigma}^{\dagger}\left(\left[U^{\dagger} \partial_{0} U\right]_{\sigma \sigma^{\prime}}+i \vec{v}_{F} \cdot\left[U^{\dagger} \vec{\nabla} U\right]_{\sigma \sigma^{\prime}}\right) \chi_{\sigma^{\prime}}\right)\right] \\
& =\int D U_{\sigma \sigma^{\prime}} D a_{\mu} \exp \left[-\int d^{3} x\left(\operatorname{Tr}\left(\frac{1}{2 \tilde{g}}\left|\left(\partial_{\mu}-i a_{\mu} \tau^{3}\right) U^{\dagger}\right|^{2}\right)\right.\right. \\
& -i a_{0} \chi_{\sigma}^{\dagger} \tau_{\sigma \sigma^{\prime}}^{3} \chi_{\sigma^{\prime}}+\vec{a} \cdot \vec{v}_{F} \chi_{\sigma}^{\dagger} \tau_{\sigma \sigma^{\prime}}^{3} \chi_{\sigma^{\prime}} \\
& \left.\left.-\frac{\tilde{g}}{2}\left|\chi_{\sigma}^{\dagger} \tau_{\sigma \sigma^{\prime}}^{3} \chi_{\sigma^{\prime}}\right|^{2}+\frac{\tilde{g}}{2}\left|\vec{v}_{F} \chi_{\sigma}^{\dagger} \tau_{\sigma \sigma^{\prime}}^{3} \chi_{\sigma^{\prime}}\right|^{2}\right)\right]
\end{aligned}
$$

Combining Eq. (3) and Eq. (5), we reach an effective field theory for the quantum phase transition in the Kondo lattice model

$$
\begin{aligned}
& Z=\int D \chi_{\sigma} D U_{\sigma \sigma^{\prime}} D a_{\mu} e^{-S_{c}-S_{m}}, \\
& S_{c}=\int d^{3} x\left[\chi _ { \sigma } ^ { \dagger } \left(\left[\partial_{0}-i A_{0}\right] \delta_{\sigma \sigma^{\prime}}-i a_{0} \tau_{\sigma \sigma^{\prime}}^{3}\right.\right. \\
& \left.+\vec{v}_{F} \cdot\left(\left[i \vec{\nabla}+\vec{k}_{F}+\vec{A}\right] \delta_{\sigma \sigma^{\prime}}+\vec{a} \tau_{\sigma \sigma^{\prime}}^{3}\right)\right) \chi_{\sigma^{\prime}} \\
& \left.-\frac{\tilde{g}}{2}\left|\chi_{\sigma}^{\dagger} \tau_{\sigma \sigma^{\prime}}^{3} \chi_{\sigma^{\prime}}\right|^{2}+\frac{\tilde{g}}{2}\left|\vec{v}_{F} \chi_{\sigma}^{\dagger} \tau_{\sigma \sigma^{\prime}}^{3} \chi_{\sigma^{\prime}}\right|^{2}\right] \\
& +\frac{2 \sqrt{2 d} a^{d-1} J_{K}}{c \tilde{g}} \int_{0}^{c \beta} d x_{0} \sum_{k} \chi_{k \sigma^{\dagger}}^{\dagger} \tau_{\sigma \sigma^{\prime}}^{3} \chi_{k+Q \sigma^{\prime}}, \\
& S_{m}=\int d^{3} x\left[\operatorname{Tr}\left(\frac{1}{2 \tilde{g}}\left|\left(\partial_{\mu}-i a_{\mu} \tau^{3}\right) U^{\dagger}\right|^{2}\right)\right] .
\end{aligned}
$$

The compact $\mathrm{U}(1)$ gauge field $a_{\mu}$ guarantees the $U_{a}(1)$ local gauge symmetry, where the gauge field is transformed into $a_{\mu}^{\prime}=a_{\mu}+\partial_{\mu} \vartheta$ under the transformations in Eq. (4). From Eq. (6) the bosonic spinons $U_{\sigma \sigma^{\prime}}^{\dagger}$ and the spinless fermions $\chi_{\sigma}$ can be considered to carry an internal charge $e_{a}$ associated with the internal $\mathrm{U}(1)$ gauge field $a_{\mu}$. Especially, the spinless fermions have not only the internal charge $e_{a}$ but also the real electric charge $e_{A}$. The kinetic energy of the $\mathrm{SU}(2)$ matrix $U^{\dagger}$ can be expressed into the familiar $C P^{1}$ representation, $(1 / 2 \tilde{g}) \operatorname{Tr}\left(\left|\left(\partial_{\mu}-i a_{\mu} \tau^{3}\right) U^{\dagger}\right|^{2}\right)=(1 / \tilde{g})\left|\left(\partial_{\mu}-i a_{\mu}\right) z_{\sigma}\right|^{2}$.

We would like to emphasize that Eq. (6) is just another representation of $\mathrm{Eq}$. (3) derived from $\mathrm{Eq}$. (2) via the gauge transformation $\chi_{\sigma}=U_{\sigma \sigma^{\prime}}^{\dagger} c_{\sigma^{\prime}}$. For this rewriting to be physically meaningful beyond a mathematical derivation, there should exist the deconfined quantum criticality of the nonlinear $\sigma$ model allowing deconfined bosonic spinons at the quantum critical point. In other words, at least the nonlinear $\sigma$ model in the effective field theory Eq. (6) should be stable in the renormalization group sense. Fortunately, this deconfined quantum criticality was shown to exist in Refs. [1, 2] by a renormalization group analysis, as mentioned before. Based on the exact transformation and the existence of the deconfined quantum criticality, we discuss the quantum critical point between an antiferromagnetic metal and a heavy fermion liquid from Eq. (6). When the deconfinement of bosonic spinons is not allowed owing to instanton effects of compact $\mathrm{U}(1)$ gauge fields $a_{\mu}[1,2]$, the effective field theory Eq. (6) becomes unstable in the present decoupling scheme and thus, another effective theory necessarily results. This indeed happens away from the quantum critical point, i.e., in an antiferromagnetic metal and a heavy fermion liquid, as will be discussed.

The gauge transformation introduced for solving the Kondo coupling term in Eq. (1) may be still suspected to be unnatural although similar decoupling schemes have been utilized in Refs. [11, 12]. In this respect it is necessary to understand the present methodology more deeply by comparing this with other well studied ones. A good example is a $d$-wave BCS theory for superconductivity of high $T_{c}$ cuprates 20, 21]. In the context of $d$-wave superconductivity the coupling term of $|\Delta| e^{i \phi} c_{\uparrow} c_{\downarrow}$ between Cooper pairs and electrons plays the same role as the Kondo coupling term of $\vec{n} \cdot c_{\sigma}^{\dagger} \vec{\tau}_{\sigma \sigma^{\prime}} c_{\sigma^{\prime}}$ between spin fluctuations and conduction electrons. Here $|\Delta|$ and $\phi$ are the amplitude and phase of Cooper pair fields. In order to solve this coupling term several kinds of gauge transformations are introduced 20, 21]. In these decoupling schemes critical phase fluctuations of Cooper pairs screen out charge degrees of freedom of electrons, causing electrically neutral but spinful electrons called "spinons". As a result the phase factor disappears in the coupling term when it is rewritten in terms of spinons. Instead, this coupling effect appears as current-current interactions of neutral spinons and phase fields of Cooper pairs in the kinetic energy of electrons. Depending on the gauge transformations, $Z_{2}$ [20] or U(1) 21] gauge fields are obtained. In this respect the present gauge transformation naturally extends the methodology of charge U(1) symmetry in the context of superconductivity to that of spin SU(2) symmetry in the context of antiferromagnetism.

As mentioned earlier, the $\mathrm{O}(3)$ nonlinear $\sigma$ model $S_{m}$ in Eq. (6) exhibits the continuous quantum phase transition at the nonzero critical spin stiffness $g_{c}^{-1}[14,15]$. 
Remember that the spin stiffness can be controlled by the Kondo coupling constant $J_{K}$. We define the critical Kondo coupling $J_{K}^{c}$ leading to the critical spin stiffness $g_{c}$. In the case of $\tilde{g}<g_{c}\left(J_{K}<J_{K}^{c}\right)$ the condensation of bosonic spinons occurs, $<U_{\sigma \sigma^{\prime}}^{\dagger}>\neq 0\left(<z_{\sigma}>\neq 0\right)$, resulting in antiferromagnetism. The spinon condensation leads the gauge field $a_{\mu}$ to be massive (Anderson-Higgs mechanism). In the context of gauge theories this phase corresponds to the Higgs-confinement phase 22, 23. The condensed bosonic spinons $U_{\sigma \sigma^{\prime}}$ are confined with the spinless fermions $\chi_{\sigma^{\prime}}$ to make the original conduction electrons $c_{\sigma}$, i.e., $U_{\sigma \sigma^{\prime}} \chi_{\sigma^{\prime}} \rightarrow c_{\sigma}$. Performing the unitary gauge $a_{\mu} \tau^{3}=a_{\mu}^{\prime} \tau^{3}-i U \partial_{\mu} U^{\dagger}$ and the gauge transformation $\chi_{\sigma}=U_{\sigma \sigma^{\prime}}^{\dagger} c_{\sigma^{\prime}}$ in Eq. (6), and integrating over the massive gauge field $a_{\mu}^{\prime}$, we obtain the following field theory for the conduction electrons in the presence of antiferromagnetism $\left(\vec{n} \cdot \vec{\tau}=\tau^{3}\right)$,

$$
\begin{aligned}
& S_{c}=\int d^{3} x\left[c_{\sigma}^{\dagger}\left(\left[\partial_{0}-i A_{0}\right]+\vec{v}_{F} \cdot\left[i \vec{\nabla}+\vec{k}_{F}+\vec{A}\right]\right) c_{\sigma}\right] \\
& +\frac{2 \sqrt{2 d} a^{d-1} J_{K}}{c \tilde{g}} \int_{0}^{c \beta} d x_{0} \sum_{k} c_{k \sigma}^{\dagger} \tau_{\sigma \sigma^{\prime}}^{3} c_{k+Q \sigma^{\prime}} .
\end{aligned}
$$

Deep inside the antiferromagnetism, the staggered Zeeman term would vanish owing to $J_{K} \rightarrow 0$ in the renormalization group sense. Thus, usual Fermi liquid physics is recovered. This phase is an antiferromagnetic metal, where the antiferromagnetism and Fermi liquid physics are nearly separated except the staggered Zeeman term.

Approaching the quantum critical point $\tilde{g} \rightarrow g_{c}\left(J_{K} \rightarrow\right.$ $\left.J_{K}^{c}\right)$, critical antiferromagnetic fluctuations of the low energy localized spin variable $\vec{n}$ would fractionalize into critical bosonic spinons $z_{\sigma}\left(U_{\sigma \sigma^{\prime}}^{\dagger}\right)[1,[2,[3]$. An important point is that owing to the nonzero critical coupling the bosonic spinons are expected to screen out the spin degrees of freedom of the conduction electrons $c_{\sigma}$, making the spinless fermions (Kondo resonances) $\chi_{\sigma}=U_{\sigma \sigma^{\prime}}^{\dagger} c_{\sigma^{\prime}}$. The internal charge $e_{a}$ would be deconfined owing to critical fluctuations of the bosonic spinons 1, 2, 3]. Furthermore, dissipative dynamics of the gauge field arising from the contribution of non-relativistic fermions $\chi_{\sigma}$ in the presence of the Fermi surface 10, 13, 24, 25, 26, 27, 28. would increase the tendency of deconfinement 9, 29]. Thus, both the critical spinless fermions and bosonic spinons would appear at the quantum critical point. The resulting critical field theory is obtained to be from Eq. (6)

$$
\begin{aligned}
& S_{Q C P}=\int d^{3} x\left[\chi _ { \sigma } ^ { \dagger } \left(\left[\partial_{0}-i A_{0}\right] \delta_{\sigma \sigma^{\prime}}-i a_{0} \tau_{\sigma \sigma^{\prime}}^{3}\right.\right. \\
& \left.\left.+\vec{v}_{F} \cdot\left(\left[i \vec{\nabla}+\vec{k}_{F}+\vec{A}\right] \delta_{\sigma \sigma^{\prime}}+\vec{a} \tau_{\sigma \sigma^{\prime}}^{3}\right)\right) \chi_{\sigma^{\prime}}\right] \\
& +\frac{2 \sqrt{2 d} a^{d-1} J_{K}}{c \tilde{g}} \int_{0}^{c \beta} d x_{0} \sum_{k} \chi_{k \sigma}^{\dagger} \tau_{\sigma \sigma^{\prime}}^{3} \chi_{k+Q \sigma^{\prime}} \\
& +\int d^{3} x\left[\operatorname{Tr}\left(\frac{1}{2 \tilde{g}}\left|\left(\partial_{\mu}-i a_{\mu} \tau^{3}\right) U^{\dagger}\right|^{2}\right)\right] .
\end{aligned}
$$

In the above we ignored the local interactions since they are irrelevant at the quantum critical point. This critical field theory is expected to show non-Fermi liquid physics owing to long range gauge interactions $10,13,24,25,26$, 27, 28]. Critical fluctuations of the bosonic spinons result in the non-Maxwell kinetic energy of the gauge field, $\frac{N_{z}}{16}(\partial \times a) \frac{1}{\sqrt{-\partial^{2}}}(\partial \times a)$, where $N_{z}$ is the flavor number of the bosonic spinons, here $N_{z}=2[30]$. This leads to the following effective action

$$
\begin{aligned}
& S_{Q C P}=\int d^{3} x\left[\chi _ { \sigma } ^ { \dagger } \left(\left[\partial_{0}-i A_{0}\right] \delta_{\sigma \sigma^{\prime}}-i a_{0} \tau_{\sigma \sigma^{\prime}}^{3}\right.\right. \\
& \left.+\vec{v}_{F} \cdot\left(\left[i \vec{\nabla}+\vec{k}_{F}+\vec{A}\right] \delta_{\sigma \sigma^{\prime}}+\vec{a} \tau_{\sigma \sigma^{\prime}}^{3}\right)\right) \chi_{\sigma^{\prime}} \\
& \left.+\frac{N_{z}}{16}(\partial \times a) \frac{1}{\sqrt{-\partial^{2}}}(\partial \times a)\right] \\
& +\frac{2 \sqrt{2 d} a^{d-1} J_{K}}{c \tilde{g}} \int_{0}^{c \beta} d x_{0} \sum_{k} \chi_{k \sigma}^{\dagger} \tau_{\sigma \sigma^{\prime}}^{3} \chi_{k+Q \sigma^{\prime}}
\end{aligned}
$$

In the absence of the last Zeeman term this effective action was shown to give $C_{v} \sim T \ln T$ and $\sigma \sim T^{-5 / 3}$ in two dimensions, where $C_{v}$ and $\sigma$ are specific heat and conductivity, respectively, and $T$, temperature 13]. Remember that in the case of the Maxwell kinetic energy the specific heat is given by $C_{v} \sim T^{2 / 3}$ in two dimensions 10,24 and $C_{v} \sim T \ln T$ in three dimensions 10, 27, 28]. The conductivity is shown to be $\sigma \sim T^{-4 / 3}$ in two dimensions and $\sigma \sim T^{-5 / 3}$ in three dimensions $10,25,26$, in the case of the Maxwell kinetic energy. We can see that the unusual dynamics of the gauge field described by the non-Maxwell kinetic energy results in "three dimensional effect" 13 ]. The non-Fermi liquid physics is not captured in the antiferromagnetic metal, where gauge fluctuations are suppressed via the Anderson-Higgs mechanism. The presence of the staggered Zeeman term would not give rise to qualitative changes in the non-Fermi liquid physics. This term is expected to make the $\chi_{\sigma}$ band flatter, renormalizing the Fermi velocity $v_{F}$ and the Fermi momentum $k_{F}$. A little heavier $\chi_{\sigma}$ fields would arise from the Zeeman term. $C_{v} \sim T \ln T$ and $\sigma \sim T^{-5 / 3}$ are expected to remain at the deconfined quantum critical point.

In the case of $\tilde{g}>g_{c}\left(J_{K}>J_{K}^{c}\right)$ the bosonic spinons are gapped, $<U_{\sigma \sigma^{\prime}}^{\dagger}>=0\left(<z_{\sigma}>=0\right)$. Deep inside the quantum disordered paramagnetism, the spin stiffness $g^{-1}$ would vanish in the renormalization group sense 14]. The vanishing spin stiffness gives rise to one problem that the strength of local interactions in Eq. (6) goes to infinity. Infinitely strong interactions are expected to suppress fluctuations of the $\chi_{\sigma}$ fermions. In order to treat the infinitely strong local interactions, we perform the Hubbard-Stratonovich transformation to obtain $i \alpha_{0} \chi_{\sigma}^{\dagger} \tau_{\sigma \sigma^{\prime}}^{3} \chi_{\sigma^{\prime}}+i \vec{\alpha} \cdot \vec{v}_{F} \chi_{\sigma}^{\dagger} \tau_{\sigma \sigma^{\prime}}^{3} \chi_{\sigma^{\prime}}$, where $\alpha_{\mu}$ is an auxiliary field. Integration over the $\alpha_{\mu}$ field leads to the local constraint of $\chi_{\sigma}^{\dagger} \tau_{\sigma \sigma^{\prime}}^{3} \chi_{\sigma^{\prime}}=0$. This local constraint makes the gauge field $a_{\mu}$ decouple to the spinless fermions. Another way to say this is that the $\chi_{\sigma}$ fields do not screen out the internal charge $e_{a}$ owing to the local constraint. Only the Maxwell kinetic energy is available, resulting in 
TABLE I: Quantum phase transition in the Kondo lattice model

\begin{tabular}{cccc}
\hline & Antiferromagnetic Metal & Quantum Critical Point & Heavy Fermion Liquid \\
& $\tilde{g}<g_{c}$ & $\tilde{g}=g_{c}$ & $\tilde{g}>g_{c}$ \\
\hline Order Parameter & $<U_{\sigma \sigma^{\prime}}^{\dagger}>\neq 0\left(<z_{\sigma}>\neq 0\right)$ & & $\left\langle U_{\sigma \sigma^{\prime}}^{\dagger}>=0\left(<z_{\sigma}>=0\right)\right.$ \\
Internal Charge $\left(e_{a}\right)$ & $U_{\sigma \sigma^{\prime}} \chi_{\sigma^{\prime}} \rightarrow c_{\sigma}$ & $c_{\sigma} \rightarrow U_{\sigma \sigma^{\prime}} \chi_{\sigma^{\prime}}$ & $c_{\sigma} \rightarrow U_{\sigma \sigma^{\prime}} \chi_{\sigma^{\prime}}$ \\
& $z_{\sigma}^{\dagger} \tau_{\sigma \sigma^{\prime}}^{ \pm} z_{\sigma^{\prime}} \rightarrow \pi^{ \pm}$ & & $z_{\sigma}^{\dagger} \vec{\tau}_{\sigma \sigma^{\prime}} z_{\sigma^{\prime}} \rightarrow \vec{n}$ \\
& Confinement of $e_{a}$ & Deconfinement of $e_{a}$ & Confinement of $e_{a}$ \\
Gapless Excitations & $\pi^{ \pm}, c_{\sigma}$ & $U_{\sigma \sigma^{\prime}}^{\dagger}\left(z_{\sigma}\right), a_{\mu}, \chi_{\sigma}$ & $\chi_{\sigma}$ \\
\hline
\end{tabular}

confinement of gapped bosonic spinons. Gapped paramagnons are expected to arise from the confinement. The resulting low energy action is given by

$$
\begin{aligned}
& S_{c}=\int d^{3} x\left[\chi_{\sigma}^{\dagger}\left(\left[\partial_{0}-i A_{0}\right]+\vec{v}_{F} \cdot\left[i \vec{\nabla}+\vec{k}_{F}+\vec{A}\right]\right) \chi_{\sigma}\right] \\
& +\frac{2 \sqrt{2 d} a^{d-1} J_{K}}{c \tilde{g}} \int_{0}^{c \beta} d x_{0} \sum_{k} \chi_{k \sigma}^{\dagger} \tau_{\sigma \sigma^{\prime}}^{3} \chi_{k+Q \sigma^{\prime}}
\end{aligned}
$$

This action describes Fermi liquid physics of the $\chi_{\sigma}$ fields under staggered magnetic fields. In the strong Kondo coupling phase $J_{K}>>J_{K}^{c}$ the Zeeman energy gap would be very large. This leads the $\chi_{\sigma}$ band to be much flat, resulting in heavy fermions $\chi_{\sigma}$. In this respect this phase is a heavy fermion liquid for the Kondo resonances $\chi_{\sigma}$. It should be noted that the present heavy fermion liquid differs from the usual one in that there is no analogous excitation with the $\chi_{\sigma}$ field in the usual heavy fermion liquid 10, 13]. A deeper analysis is required to discriminate these two heavy fermion liquids. We summarize the quantum phase transition from an antiferromagnetic metal to a heavy fermion liquid in Table I.

Our present approach has some analogies with that of Ref. [12] in that bosonic spinons are utilized for impurity spins and spinless fermions are introduced for Kondo resonances. However, there are two important differences between our approach and theirs. A critical field theory describing deconfined quantum criticality necessarily has a new emergent gauge structure in association with fractionalized fields. Our critical field theory Eq. (8) allows
U(1) gauge fields guaranteeing the emergent U(1) gauge symmetry while the critical theory in Ref. [12] does not. Although use of the $\mathrm{U}(1)$ gauge field is not a problem, it is not clear whether the perturbative evaluations in Ref. [12] keep the new U(1) gauge symmetry in all steps. In addition, our spinless fermions have two components while the ones in Ref. [12] have only one component corresponding to the $\chi_{\uparrow}$ field in our representation for the spinless fermions. Although we don't have any idea how the presence of two components qualitatively alters the physics of one component at present, we expect, at least, quantitative changes.

Lastly, we would like to point out that Fermi surface volume continuously changes in the present description, consistent with Ref. [12]. In the present theory the staggered Zeeman term controls the band of charged fermions. Since the staggered magnetic field continuously varies across the quantum critical point, the fermion band smoothly changes, thus resulting in continuous transformation of Fermi surface volume.

In the present paper we claimed that the continuous quantum phase transition in the Kondo lattice model can be realized by the deconfined quantum criticality. Our critical field theory in Eq. (6) not only explains nonFermi liquid physics near the quantum critical point but also recovers Fermi liquid physics away from the quantum critical point.

We would like to thank Dr. Kim, Mun-Dae for helpful discussions associated with his recent work in the Kondo lattice model 31].
[1] T. Senthil, A. Vishwanath, L. Balents, S. Sachdev, and M. P. A. Fisher, Science 303, 1490 (2004); T. Senthil, L. Balents, S. Sachdev, A. Vishwanath, and M. P.A. Fisher, Phys. Rev. B 70, 144407 (2004).

[2] Ki-Seok Kim, Phys. Rev. B 72, 035109 (2005).

[3] D. Yoshioka, G. Arakawa, I. Ichinose, and T. Matsui, Phys. Rev. B 70, 174407 (2004); G. Arakawa, I. Ichinose, T. Matsui, and K. Sakakibara, Phys. Rev. Lett. 94, 211601 (2005).

[4] P. A. Lee, N. Nagaosa, and X.-G. Wen, cond-mat/0410445 and references therein.

[5] W. Rantner and X.-G. Wen, cond-mat/0105540 W. Rantner and X.-G. Wen, Phys. Rev. B 66, 144501 (2002).

[6] M. Hermele, T. Senthil, M. P. A. Fisher, P. A. Lee, N. Nagaosa, and X.-G. Wen, Phys. Rev. B 70, 214437 (2004).
[7] F. S. Nogueira and H. Kleinert, cond-mat/0501022

[8] Ki-Seok Kim, Phys. Rev. B 70, 140405(R) (2004); KiSeok Kim, Phys. Rev. B 72, 014406 (2005).

[9] Ki-Seok Kim, cond-mat/0502652

[10] T. Senthil, M. Vojta, and S. Sachdev, Phys. Rev. B 69, 035111 (2004); references therein; T. Senthil, S. Sachdev, and M. Vojta, Phys. Rev. Lett. 90, 216403 (2003).

[11] P. Coleman, C. Pepin, Q. Si (Rice), and R. Ramazashvili, Journal of Physics: Condensed Matter 13, 723 (2001).

[12] C. Pepin, Phys. Rev. Lett. 94, 066402 (2005).

[13] Ki-Seok Kim, Phys. Rev. B 71, 205101 (2005).

[14] A. Auerbach, Interacting Electrons and Quantum Magnetism, Springer (1998).

[15] N. Nagaosa, Quantum Field Theory in Strongly Correlated Electronic Systems, Springer (1999). 
[16] G. Sierra, J. Phys. A 29, 3229 (1996).

[17] Ki-Seok Kim, cond-mat/0410231

[18] A. W. Sandvik, A. V. Chubukov, and S. Sachdev, Phys. Rev. B 51, 16483(R).

[19] Ki-Seok Kim, cond-mat/0504541

[20] T. Senthil and M. P. A. Fisher, Phys. Rev. B 62, 7850 (2000).

[21] M. Franz and Z. Tesanovic, Phys. Rev. Lett. 87, 257003 (2001); This was pointed out by prof. Tesanovic.

[22] E. Fradkin, S. H. Shenker, Phys. Rev. D 19, 3682 (1979).

[23] N. Nagaosa and P. A. Lee, Phys. Rev. B 61, 9166 (2000).

[24] Alexei M. Tsvelik, Quantum Field Theory in Condensed Matter Physics (Ch. 12), Cambridge University Press (1995).
[25] P. A. Lee and N. Nagaosa, Phys. Rev. B 46, 5621 (1992).

[26] L. B. Ioffe and G. Kotliar, Phys. Rev. B 42, 103408 (1990).

[27] T. Holstein, R. E. Norton, and P. Pincus, Phys. Rev. B 8, 2649 (1973).

[28] M. Yu. Reizer, Phys. Rev. B 40, 11571 (1989).

[29] N. Nagaosa, Phys. Rev. Lett. 71, 4210 (1993).

[30] H. Kleinert, F. S. Nogueira, and A. Sudbo, Phys. Rev. Lett. 88, 232001 (2002); H. Kleinert, F. S. Nogueira, and A. Sudbo, Nucl. Phys. B 666, 361 (2003).

[31] M. D. Kim, C. K. Kim, and J. Hong, Phys. Rev. B 68, 174424 (2003). 